\title{
A synergistic relationship between PCL and natural polymers enhances the physical properties and biological activity of scaffolds
}

Prasad Sawadkar ${ }^{1 *}$, Jeviya Mohanakrishnan ${ }^{1}$, Poojitha Rajasekar ${ }^{2}$, Benyamin Rahmani ${ }^{3}$, Nupur Kohli ${ }^{1}$, Laurent Bozec ${ }^{4}$, Elena García-Gareta ${ }^{1}$

1) Regenerative Biomaterials Group, RAFT Institute, Mount Vernon Hospital, Northwood, UK.

2) Division of Respiratory Medicine, University of Nottingham, Nottingham, UK.

3) Department of Mechanical Engineering, University College London, London, UK.

4) Faculty of Dentistry, University of Toronto, Toronto, Ontario, Canada.

*Corresponding author

Dr Prasad Sawadkar

RAFT Institute

Mount Vernon Hospital

Northwood, Middlesex,

UK, HA6 2RN

Phone +44-1923-844555

Email: prasad.sawadkar@ucl.ac.uk 


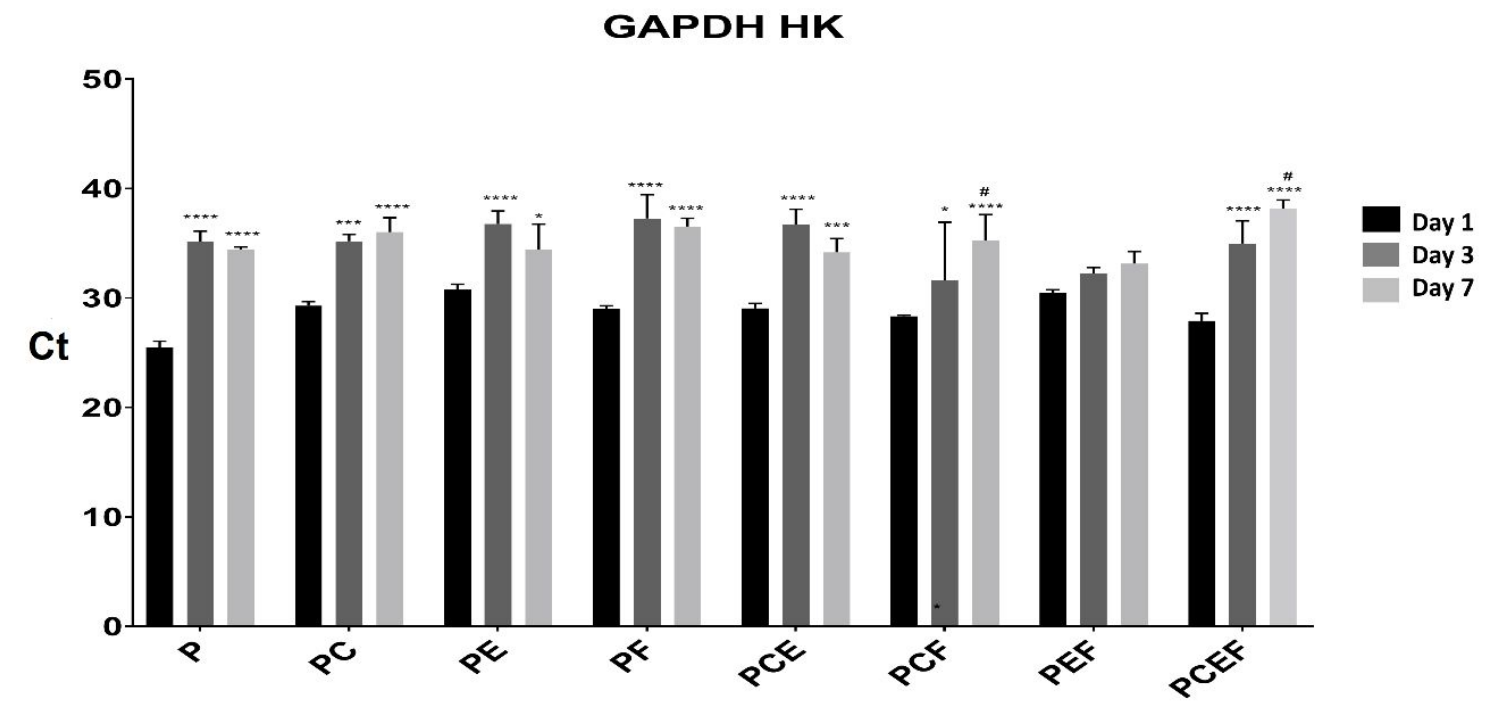

Figure S1. Expression of housekeeping gene GAPDH $(* p<0.05, * * p<0.01 * * * p<0.001$ and $* * * * p<0.0001$ to day 1 and $\# p<0.05$ to day 3 ) 


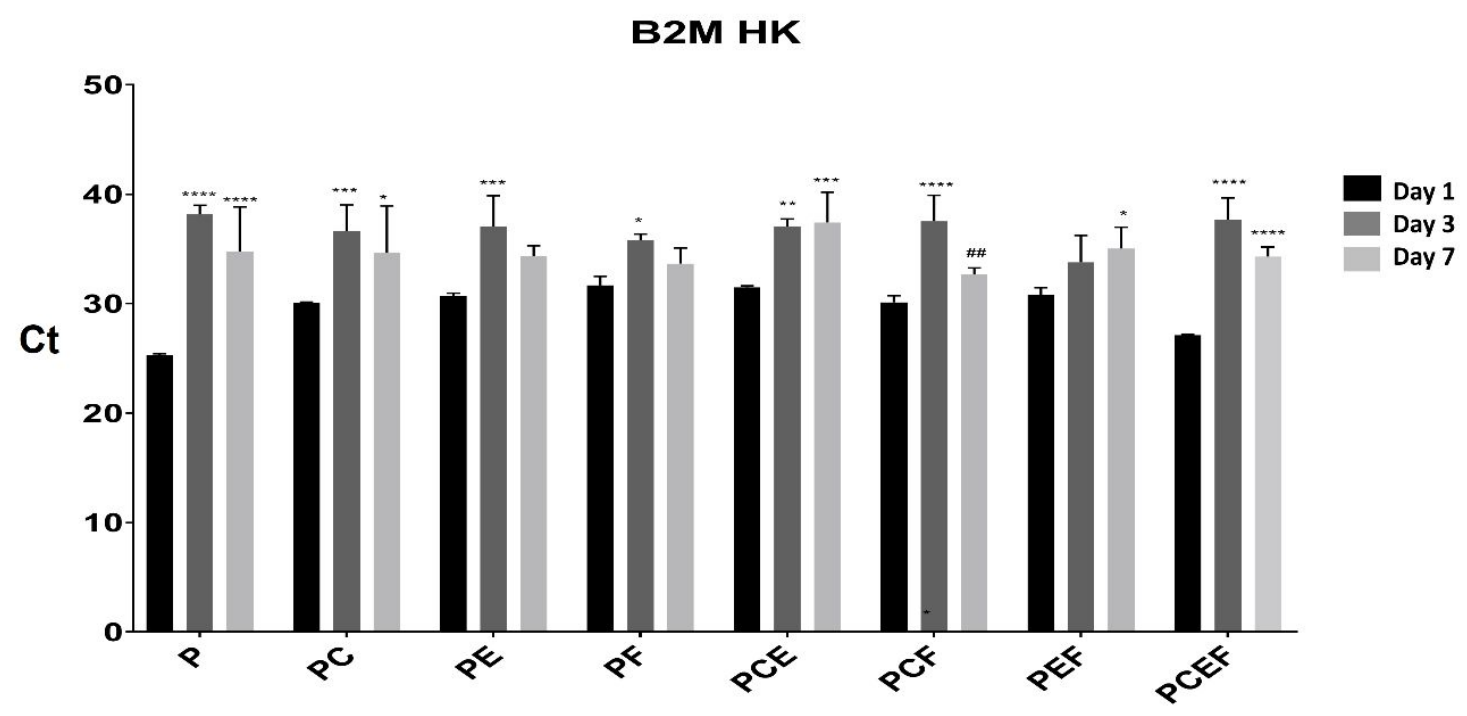

Figure S2. Expression of housekeeping gene B2M ${ }^{*} p<0.05$, $* * p<0.01 * * * p<0.001$ and **** $p<0.0001$ to day 1 and \#\# $p<0.01$ to day 3$)$ 


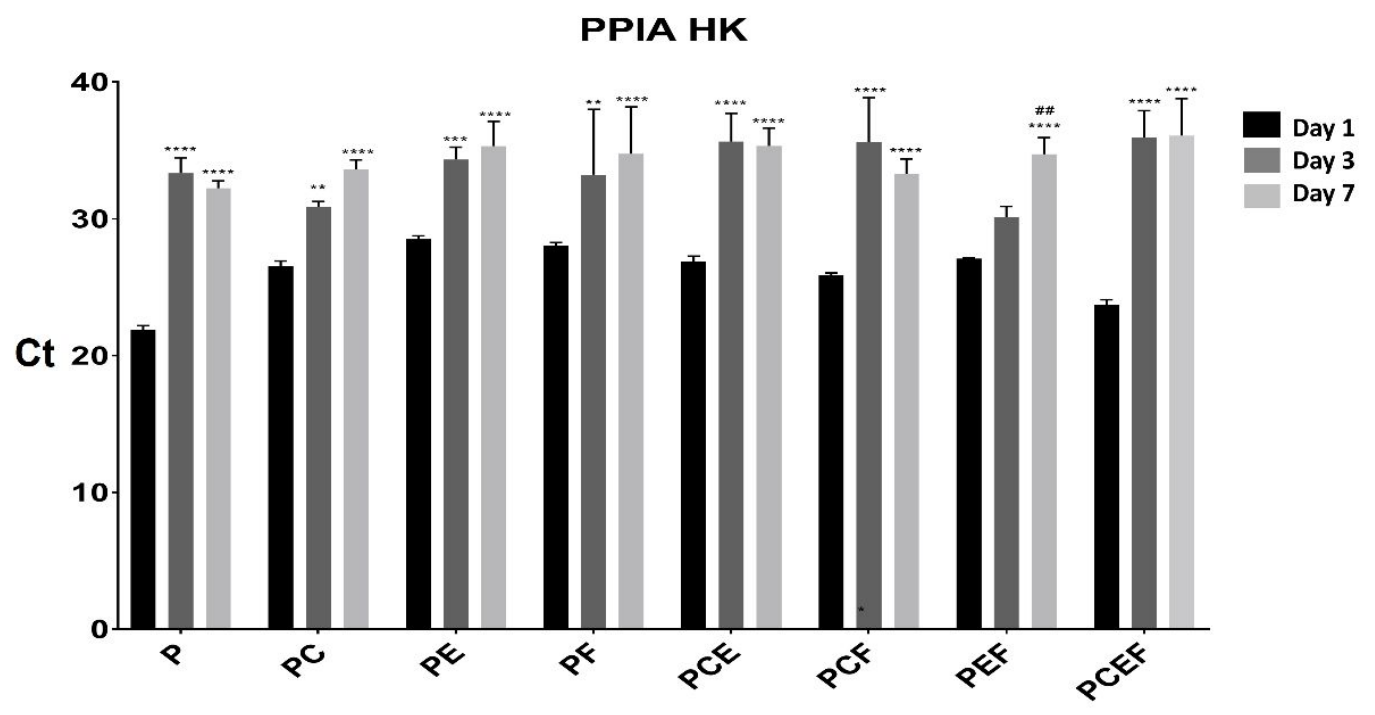

Figure S3. Expression of housekeeping gene PPIA $(* p<0.05, * * p<0.01 * * * p<0.001$ and $* * * * p<0.0001$ to day 1 and \#\# to day 3) 